\section{The CHARGE association}

We read with interest the proposal of a new autosomal recessive syndrome by Hurst et al (F Med Genet 1989;26:407-9) based on the description of two sibs with ASD, VSD, developmental delay, poor growth, microcephaly, low set, posteriorly rotated ears, and other dysmorphic features. The brother had choanal stenosis and the sister was also suspected to have choanal stenosis, but this was not proven. The authors eliminated CHARGE association because they concluded that it is a sporadic defect. However, there have been several reports of familial CHARGE association, also known as CHARGE syndrome. Metlay et $a l^{1}$ reported one such family and reviewed eight others in published reports. Of these, six appeared most likely to be autosomal dominant, two to be autosomal recessive, and one involved affected monozygotic twins and so could be either dominant or recessive. Two additional sets of affected monozygotic twins have been reported, ${ }^{2}{ }^{3}$ as well as another family that appears to represent autosomal recessive inheritance. ${ }^{4}$ Thus, CHARGE association appears to be aetiologically heterogeneous with most cases sporadic but a small yet significant percentage familial.

The boy reported by Hurst et al satisfies five of the seven major criteria for CHARGE (congenital heart disease, choanal stenosis, growth deficiency, mental deficiency, and ear anomalies) and had two other commonly reported findings (microcephaly and micrognathia). His sister meets four major criteria (congenital heart disease, growth deficiency, mental retardation, and ear anomalies), lacking only the choanal stenosis, although the authors suspected that she also had this. Choanal atresia rather than stenosis is the typical anomaly in CHARGE, but stenosis has been reported as a variant in CHARGE as have some of the dysmorphic features seen in these two patients, that is, long philtrum and high palate. ${ }^{5}$ The published photographs of the sibs both appear to show some degree of facial asymmetry with raised left eyes. Facial asymmetry is commonly observed in children with CHARGE association. ${ }^{2}$ Therefore we suggest that, rather than a new recessive syndrome, the brother and sister appear to have CHARGE syndrome. No ophthalmological or audiological examinations were reported; these studies are strongly indicated in any child with CHARGE syndrome.

\section{G Bialer, W T BROWN} Division of Genetics, Department of Pediatrics, North Shore University Hospital, Cornell University Medical Center, 300 Community Drive, Manhasset, New York 11030, USA.

1 Metlay LA, Smythe PS, Miller ME. Familial CHARGE syndrome: clinical report with autopsy findings. $\mathrm{Am} \mathcal{J}$ Med Genet 1987;26:577-81.

2 Oley CA, Baraitser M, Grant DB. A reappraisal of the CHARGE association. f Med Genet 1988;25:147-56.

3 Chestler RJ, France TD. Ocular findings in CHARGE syndrome. Ophthalmology 1988;95:1613-9.

4 Pergament E, Halperin D, Verlinsky Y, Friedman K. Microphthalmia, coloboma, cleft palate, ear malformations, ptosis and congenital heart disease: a new malformation syndrome. Birth Defects Conference Abstracts, Birmingham, AL, 1982:233.

5 Davenport SLH, Hefner MA, Mitchell JA. The spectrum of clinical features in CHARGE syndrome. Clin Genet 1986; 29:298-310.

This letter was shown to Drs Hurst and Berry, who reply as follows.

Thank you for the opportunity to reply to the letter from Drs Bialer and Brown. After careful consideration of the points raised in the letter we do not think that the brother and sister we described have the CHARGE association.

While the CHARGE association was considered in our differential diagnosis, we do not consider that they have sufficient features of that association to make the diagnosis. In particular there were no colobomata, the ears, though posteriorally rotated, were structurally normal, they were not deaf, there was no facial asymmetry or VIIth nerve palsy, the heart lesion is not that which is most commonly found in CHARGE (Fallot or conotruncal lesions), and there were no other commonly associated malformations such as tracheooesophageal fistula.

There are many hundreds of children known to have the CHARGE association but only a few reported sib pairs. In the unusual situation of affected brothers and sisters we consider it necessary for both to have the full clinical picture to make a diagnosis of the CHARGE association.
However, there will remain diagnostic difficulty until the underlying gene defect in CHARGE association is identified.

JANE HURST
Department of Clinical Genetics,
The Hospital for Sick Children,
Great Ormond Street,
London WCIN 3fH.
CAROLINE BERRY
Paediatric Research Unit,
Guy's Hospital,
London SEl 9RT.

The Moebius syndrome: aetiology, incidence of mental retardation, and genetics

Kumar, in his summary of the Moebius syndrome $(\mathcal{F}$ Med Genet 1990;27: 122-6), has made certain contentions with regard to the aetiology, incidence of mental retardation, and genetic transmission of this syndrome which require further examination.

The aetiology of this syndrome has been debated since its original description. Arguments have centred around questions of whether the disorder is primarily degenerative or dysplastic, and the location of the pathology, whether in the brain stem, the peripheral nerves, or the muscle of the face or eye. In addition, to overlap with the hypoglossia-hypodactyly, ankyloglossia, and Charlie $M$ syndromes, there is also association with the Hanhart syndrome. A recent study into the aetiology of the Moebius syndrome has indicated significant events of pregnancy in eight of 15 cases, including hyperthermia, previous uterine surgery, electric shock, failed abortion, prolonged rupture of the membranes, and alcohol abuse. ${ }^{1}$ The association with hyperthermia during pregnancy has been confirmed in another study. ${ }^{2}$ In a rat animal model, uterine artery occlusion, handling the uterine vessels, and uterine handling were associated with bilateral brain stem ischaemic lesions and peripheral limb deficiency. ${ }^{3}$

In this animal study the mothers were treated on day 16 of pregnancy, after organogenesis and lesions were seen in the brain stem and pons. Similar lesions have been seen in a rat animal model of cocaine abuse, the 